\title{
Providing Services on Demand By User Action Modeling on Smart Phones
}

Kumar Vishal

International Institute of

Information Technology,

Hyderabad

Romil Bansal

International Institute of

Information Technology,

Hyderabad

Anoop M. Namboodiri

International Institute of

Information Technology,

Hyderabad

\section{V. Jawahar}

International Institute of

Information Technology,

Hyderabad

Permission to make digital or hard copies of part or all of this work for persona or classroom use is granted without fee provided that copies are not made or distributed for profit or commercial advantage and that copies bear this notice and the full citation on the first page. Copyrights for third-party components of this work must be honored. For all other uses, contact the Owner/Author. Copyright is held by the owner/author(s).

UbiComp'14 Adjunct, September 13-17, 2014, Seattle, WA, USA

Copyright 2014 ACM 978-1-4503-3047-3/14/09..\$15.00

http://dx.doi.org/10.1145/2638728.2638779

\begin{abstract}
We propose a novel approach to schedule services like $\mathrm{Wi}-\mathrm{Fi}$ and $3 \mathrm{G}$ on smartphones. Using Wi-Fi as an example, we show that intelligent scheduling based on a user's activity level leads to lower power consumption without adversely affecting the user experience. Data from various sensors is used to model and predict a user's activity, which is then used to schedule the wireless services.
\end{abstract}

\section{Author Keywords}

Energy Efficiency; User Activity Modeling; Smartphone

\section{ACM Classification Keywords}

I.5.4 [Pattern Recognition]: Applications

\section{Introduction}

Services like $\mathrm{Wi}-\mathrm{Fi}$ and $3 \mathrm{G}$ continue to consume significant amount of battery on smartphones. Hence, reducing the power consumption by these processes could help in huge power savings. In this work, we model and monitor the user activity level to decide when the wireless data module should be turned off for maximal energy saving without compromising the user experience. The problem is formulated as a classification problem, where $\mathrm{Wi}-\mathrm{Fi}$ is scheduled based on the predicted smartphone usage level by the user in the next time window. The 


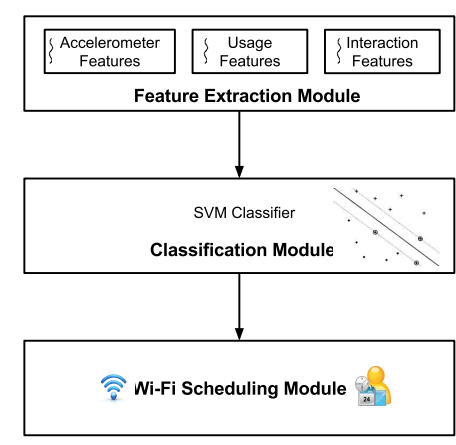

Figure 1: Steps in the algorithm with data flow. Feature extraction module extracts accelerometer, usage and interaction based features.

Classification module predicts the user activity level using an SVM Classifier and Wi-Fi Scheduling Module controls the Wi-Fi activity based on the predicted output. major contributions can be summarized as follows.

- Activity Modeling on Smartphone: We propose a power-efficient approach to modeling the user's activity level on smartphone device using accelerometer sensors and other parameters that are easily available on most smartphones.

- Wi-Fi Scheduling with Activity Level: An approach to schedule Wi-Fi service for better energy management is proposed.

Although the approach is tested with Wi-Fi in this work, it is readily applicable to other data services such as $3 \mathrm{G}$.

\section{Related Work}

Several works have been published that do activity and gesture recognition with the help of accelerometer sensor data. Sun et al. [1] proposed a mechanism to classify physical activities of the bearer of the device using accelerometer data with varying positions and orientations. We utilize other factors such as CPU and RAM usage in addition to accelerometer data to model the nature of user activity. Zhuang et al. [2] looked at the problem of energy-efficient location sensing on a smartphone. They tried to find the location using substitution, suppression, piggybacking and adaptation methods. Unlike the above works that tries to do a specific task in an efficient manner, we use a combination of activity recognition and usage features for intelligent scheduling the Wi-Fi service, which in turn leads to low power consumption by device.

\section{Activity Modeling and Scheduler Design}

The proposed framework consists of three main modules: feature extraction, classification and wireless scheduler. Figure 1 shows a schematic diagram of our approach. The goal of the proposed model is to predict the activity level of the user in the immediate future (next half and hour) into one of the four classes: very low $\left(l_{0}\right)$, low $\left(l_{1}\right)$, medium $\left(l_{2}\right)$, and high $\left(l_{3}\right)$. If we are able to predict this accurately, one can schedule wireless services accordingly to reduce the power consumption of the device. However, we need to make this prediction using parameters that are readily accessible to the system. Moreover, the collection of these parameters and prediction process should consume significantly lesser power than what is saved.

\section{Feature Extraction Module}

There are several parameters that would really help us in predicting the need for wireless services such as usage of specific applications, internal states of these applications, physical location of the user, type of interactions, calender schedule of the user, etc. However, these parameters are either too specific to be applicable to all mobile phone users or are too difficult or expensive to capture. We therefore investigate the use of a few generic and easily accessible parameters for the prediction. The parameters or features are divided into three categories- accelerometer based, system usage based and interaction based features. These features are explained in details below.

Accelerometer Based Features: Accelerometer readings are indication of the physical activity of the user, which in turn may suggest the phone usage. For example, if the user is running or sleeping he is much less likely to use the phone as compare to sitting state. We consider a sequence of samples of the 3D acceleration of the device: $S=\left(S_{X}, S_{Y}, S_{Z}\right)$ along the three axes as well as the overall acceleration: $S_{\phi}=\left\|S_{X}, S_{Y}, S_{Z}\right\|$. The accelerometer data is tracked for 30 seconds every half an hour with a sampling frequency of $5 \mathrm{~Hz}$ and the following features are extracted. [1-4] : Average acceleration along $S_{X}, S_{Y}, S_{Z}$ and the $S_{\phi}$ vector, and [5-8]: their 


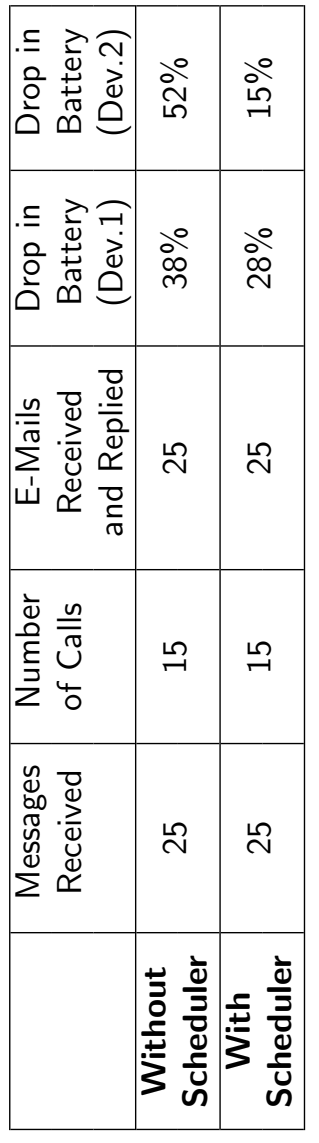

Table 1: Energy Efficiency Testing: Percentage battery dropped in Samsung Galaxy S (Dev.1) and HTC Explorer

(Dev.2) during controlled tests. standard deviations within the 30 seconds. $[9-14]$ : Correlation among each pair of $S_{X}, S_{Y}, S_{Z}$ and the $S_{\phi}$ vector, which indicates the strength of dependence between the two corresponding signals. $[15-18]$ : Fourier energy for $S_{X}, S_{Y}, S_{Z}$ and the $S_{\phi}$ vector. The energy is the normalized sum of all the squared DFT component magnitudes except the DC component. [19-22] : Fourier entropy for $S_{X}, S_{Y}, S_{Z}$ and $S_{\phi}$ vectors. Fourier entropy is the normalized information present in the DFT of the signal, excluding the DC component.

System Usage Based Features: System Usage Based features tells how extensively the phone is being utilized. The two factors that we measure are the average CPU utilization and the average memory used by the applications in the smartphone.

Interaction Based Features: Interaction based features tells how frequently user is using his phone. These include the fraction of time for which the screen was on in the recent past and the frequency of interaction indicated by the number of times the screen was been turned off and on.

All the features are extracted in parallel to get consistent data and reduce the battery consumption due to wake-lock acquisition by the application. Finally we concatenate all the features to form a final feature vector of length 30 for the classification.

\section{Classification and Scheduling Module}

Feature vector from the feature extraction module is used by SVM classifier to predict the activity label and schedule Wi-Fi for next half an hour into one of the four activity levels as mentioned before. The scheduler takes the following actions as per the classification
- $l_{0}$ : Turn off the Wi-Fi for next 30 minutes.

- $l_{1}$ : Turn off the Wi-Fi for next 25 minutes then turn it on for the following 5 minutes.

- $l_{2}$ : Turn off the Wi-Fi for next 20 minutes then turn it on for the following 10 minutes.

- $l_{3}$ : Turn on the Wi-Fi for the next 30 minutes.

To train our classifier we create an application that logs the relevant parameters from a smartphone along with the internet activity, thus giving us labeled data for learning. The application logs accelerometer readings, screen on duration, internet usage, and CPU and RAM usage of the device during every half an hour interval. The class label of an interval is decided based on the internet usage by the user within the time window. We ran the application for a total of over 6 working days on smart phones, which resulted in 310 labeled feature samples. The set contained $183 l_{0}$ samples, $67 l_{1}$ samples, $23 l_{2}$ samples and $37 l_{3}$ samples. To remove the skew in data on the classifier learned, we penalized learning errors of each class by a weight that is inversely proportional to the number of samples. Table 2 shows the penalty weights assigned to different classes while training.

\section{Implementation and Results}

We implemented the software on Android OS. The trained SVM model along with the feature extraction, classification and scheduling module is deployed on the smartphone. SVM parameters are selected using grid search and best performance is observed with $C=16$ and $\gamma=0.125$ for the RBF kernel. After each half an hour, the application starts a service to collect the data. The service runs for the duration of 30 seconds and executes the following processes.

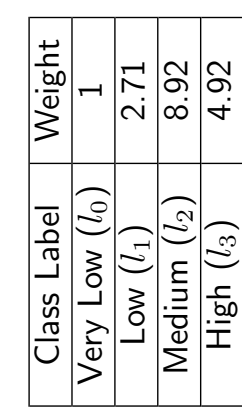

Table 2: Penalty weight assigned to different activity level while training. 


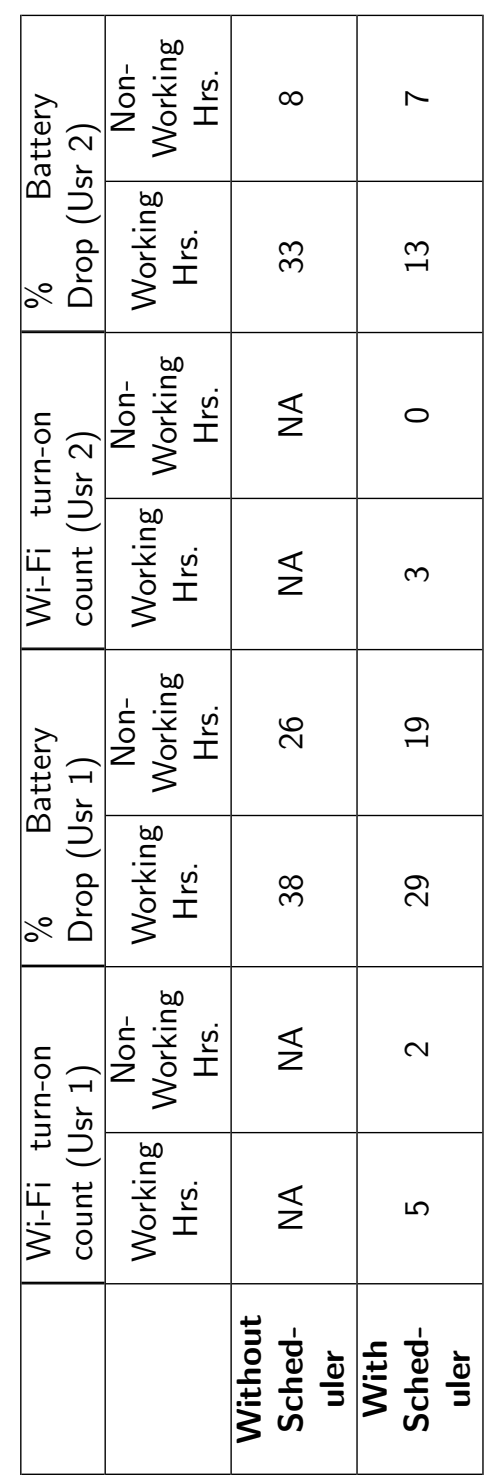

Table 3: User Happiness: Count of Number of times Wi-Fi was manually turned on.
1. Partial wake-lock is acquired to prevent smartphone from going to sleep during the process execution.

2. The required data is collected for the duration of 30 seconds. Activity label is predicted by the classification module from the collected data.

3. The Wi-Fi is scheduled for next half an hour based on the predicted label.

4. Wake-lock is released and phone is allowed to go to sleep.

We evaluated our approach based on two metrics: (i) power saved metric to measure the overall power saved by our method and (ii) user happiness metric to measure user's satisfaction with the services scheduled.

Power Savings: To evaluate the power saving by our software we took two devices each of two different models ${ }^{1}$ having the same set of applications, battery state and memory. One device of each type has our software installed, while the other does not. A predefined set of activities are performed on all the devices and drop in battery is recorded. Table 1 lists the activities carried out on the smartphone. All the activities are performed at almost same time and for the same duration. For example, the calls on all the devices are performed simultaneously for 2 minutes duration. Similarly, all the messages and emails are also send simultaneously so that the loss incurred by other processes remains same. The controlled experiment was performed for 4 hours in similar settings and the battery dissipated on each device is recorded. Our application took only $5 \mathrm{MB}$ of RAM and $101 \mathrm{~KB}$ storage, with power consumption equal to $1 \%$ of battery in 24 hours $^{2}$.

\footnotetext{
${ }^{1}$ Samsung_S_plus and HTC_Explorer

${ }^{2}$ The power consumed by the application is recorded through battery info manager on Android OS.
}

User Happiness: We conducted an experiment in which we gave our software to users and logged their battery usage in two categories: when the user is very active (working hours) and when the user is less active (non-working hours). Along with the battery usage, the number of times the Wi-Fi state is changed manually is also recorded. Each Wi-Fi switch by the user is considered as misclassification by the algorithm. The higher the number of switches by the user, the more unhappy the user is with the application. Table 3 shows a six-hour analysis of the application. We observe that application is able to conserve battery without adversely affecting the user experience significantly.

\section{Conclusions and Future Work}

We have shown that modeling user activity can help smartphones use its power more intelligently. One could further improve the results by scheduling the time interval of the application dynamically, making it more adaptive to user's activity level ${ }^{3}$.

\section{References}

[1] Sun, L., Zhang, D., Li, B., Guo, B., and Li, S. Activity recognition on an accelerometer embedded mobile phone with varying positions and orientations. In Proceedings of the 7th International Conference on Ubiquitous Intelligence and Computing, Springer-Verlag (Berlin, Heidelberg, 2010), 548-562.

[2] Zhuang, Z., Kim, K.-H., and Singh, J. P. Improving energy efficiency of location sensing on smartphones. In Proceedings of the 8th International Conference on Mobile Systems, Applications, and Services (2010), 315-330.

${ }^{3}$ This publication is an outcome of the R\&D work undertaken in the ITRA project of Media Lab Asia entitled "Virtual Assistant for Mobile Devices using Voice and Gesture Technologies" 